\title{
Integrated traditional Chinese medicine alleviates sciatica while regulating gene expression in peripheral blood
}

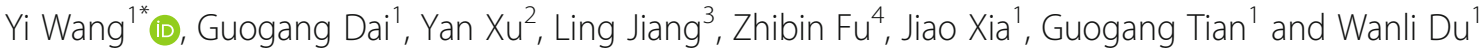

\begin{abstract}
Background: Although integrated traditional Chinese medicine (TCM) has long been indicated to be effective in the treatment of sciatica and is widely used in the management of this condition, the mechanism by which integrated TCM alleviates sciatica has not yet been fully defined, and the effect of integrated TCM on gene expression in the peripheral blood of patients with sciatica is still unknown. We performed this study to investigate the effect of integrated TCM on peripheral blood gene expression in patients with sciatica and to explore new clues for studying the mechanism of integrated TCM in alleviating sciatica.

Methods: We used a microarray to identify differentially expressed genes (DEGs) in the peripheral blood of patients with sciatica and healthy controls (DEGs-baseline), bioinformatic analysis to reveal the characteristics of DEGsbaseline, and the key genes that contribute to the gene dysregulation. A microarray was also used to identify DEGs in the peripheral blood of patients with sciatica after integrated TCM treatment compared with those at baseline, and the expression levels of DEGs were validated by qRT-PCR.
\end{abstract}

Results: We identified 153 DEGs-baseline, which included 131 upregulated genes and 22 downregulated genes. Bioinformatic analysis revealed that most of the DEGs-baseline were related to immunity and the inflammatory response and that TLR4, MMP9, MPO, CAMP, RETN, TLR5, and ILIRN were key genes involved in the dysregulation of genes in the peripheral blood of patients with sciatica. The expression levels of TLR5, IL1RN, SLC8A1, RBM20, GPER1, IL27, SOCS1, and GRTP1-AS1 were decreased in the peripheral blood of patients after integrated TCM treatment compared with that at baseline, which was accompanied by relief of pain.

Conclusion: Integrated TCM treatment relieved pain while regulating the gene expression of TLR5, IL1RN, SLC8A1, RBM20, GPER1, IL27, SOCS1, and GRTP1-AS1 in the peripheral blood of patients with sciatica. Our study provides new clues for studying the mechanism of TCM in treating sciatica.

Keywords: Sciatica, Peripheral blood, Gene expression, Traditional Chinese medicine

\footnotetext{
* Correspondence: yiwang1984@hotmail.com

${ }^{1}$ Cervicodynia/Omalgia/Lumbago/Sciatica Department 2, Sichuan Provincial Orthopedics Hospital, No. 132 West First Section First Ring Road, Wuhou District, Chengdu, Sichuan Province, China

Full list of author information is available at the end of the article
}

C C The Author(s). 2021 Open Access This article is licensed under a Creative Commons Attribution 4.0 International License, which permits use, sharing, adaptation, distribution and reproduction in any medium or format, as long as you give appropriate credit to the original author(s) and the source, provide a link to the Creative Commons licence, and indicate if changes were made. The images or other third party material in this article are included in the article's Creative Commons licence, unless indicated otherwise in a credit line to the material. If material is not included in the article's Creative Commons licence and your intended use is not permitted by statutory regulation or exceeds the permitted use, you will need to obtain permission directly from the copyright holder. To view a copy of this licence, visit http://creativecommons.org/licenses/by/4.0/ The Creative Commons Public Domain Dedication waiver (http://creativecommons.org/publicdomain/zero/1.0/) applies to the data made available in this article, unless otherwise stated in a credit line to the data. 


\section{Background}

Sciatica is characterized by neuropathic pain in the sciatic nerve, and the lifetime incidence is up to $40 \%$ [1]. Lumbar disc herniation is a major cause of sciatica and a major concern in studies about sciatica [2]. It is generally recognized that during lumbar disc herniation, mechanical compression in combination with immunity and inflammation causes sciatica. Many studies have revealed inflammatory transcriptome characteristics of degenerated discs [3-6], and many cytokines related to immunity and inflammation were activated in herniated lumbar discs [7-10]. However, since sciatica involves a focal lesion, gene expression changes in peripheral blood caused by sciatica have rarely been studied.

Many methods have been reported to improve sciatica, and $90 \%$ of acute sciatica can be effectively relieved by nonsurgical treatment [11]. Integrated traditional Chinese medicine (TCM) has long been indicated to be effective in the treatment of sciatica and is widely used in the management of this condition [12-14]. However, the mechanism by which integrated TCM relieves sciatica has not yet been fully defined. Therefore, we performed the present study to investigate the gene expression changes after integrated TCM treatment in the peripheral blood of patients with sciatica, aiming to explore new clues for studying the role of traditional Chinese medicine in alleviating sciatica.

\section{Methods}

\section{Enrollment and integrated TCM treatment}

This study was approved by the Ethics Committee of Sichuan Provincial Orthopedics Hospital, and written informed consent was obtained. From April 2018 to December 2019, 25 patients and 25 healthy volunteers were enrolled in this observational study. Patients were enrolled from those who chose to receive nonsurgical treatment with integrated TCM in our hospital. Diagnostic criteria of sciatica was set according to the guidelines of the North American Spine Society [15]: (1) a history of pain in the distribution of sciatic nerve, (2) a positive Lasegue's sign, and (3) had findings on magnetic resonance imaging of lumbar disc herniation at the L $4 / 5$ level or L5/S1 level with compression of the corresponding nerve root. The patient inclusion criteria were as follows: (1) aged between 18 and 60 years, (2) had sciatica secondary to single-level lumbar disc herniation at the L4/5 level or L5/S1 level, and (3) had no medication history within 3 months before the appointment. The exclusion criteria were as follows: (1) concomitant cauda equina syndrome, other neuropathy, other spinal diseases, infection, rheumatism, cardiovascular disease, metabolic disease, hematopoietic diseases, dementia, consciousness disturbance, or mental illness; (2) a history of surgery, congenital disease, tuberculosis, or tumors; (3) pregnancy or lactation; and (4) other medical conditions that might affect gene expression in peripheral blood. Healthy volunteers were recruited as healthy controls. All patients underwent standard integrated TCM treatment for sciatica according to our medical protocol, including electric acupuncture, massage, moxibustion, and Chinese medicine fumigation for waist circumference. The pain intensity score on the visual analogue scale (VAS, ranging from 0 to 10 , with higher scores indicating greater pain intensity) was recorded.

\section{Peripheral blood collection, RNA extraction, and microarray analysis}

Peripheral blood was collected and frozen in PAXgene tubes, and then, total RNA was extracted and purified using the PAXgeneTM Blood RNA Kit (Qiagen, Germany). An Agilent SurePrint G3 Microarray $(8 \times$ $60 \mathrm{~K})$ was used for the microarray experiment, which was performed by Shanghai Biotech Co. (Shanghai, China). These procedures were described in our previous study [16].

\section{Differentially expressed genes (DEGs)}

Two groups of DEGs were identified: DEGs in the peripheral blood of patients with sciatica and healthy controls (DEGs-baseline) and DEGs in the peripheral blood of patients with sciatica after integrated TCM treatment compared with those at baseline (DEGs-TCM).

Data from the chip scan were $\log 2$ normalized for comparative analyses. Unrecognized probes were discarded. We deleted unrecognized probes. The average value of the data from the probes corresponding to the same gene was used for analysis. Genes with an absolute fold change (FC) of $\geq 1.5$ were identified as DEGs, and the FC data were filtered by $t$ tests $(P<0.05)$. Gene expression data sets are accessible on the Gene Expression Omnibus (GEO) database (http://www.ncbi.nlm.nih.gov/ geo) under the number GSE150408 and GSE124272.

\section{Enrichment analysis of DEGs-baseline}

Enrichment analysis was performed with the web-based portal Metascape [17], which included Gene Ontology (GO) analysis of biological processes (BP), cellular components (CC), and molecular functions (MF), and Kyoto Encyclopedia of Genes and Genomes (KEGG) pathway analysis.

\section{Protein-protein interaction (PPI) network}

To identify key genes in the dysregulation of gene expression in the peripheral blood of patients at baseline and in healthy controls, we used the STRING database to construct the PPI network of DEGs-baseline with a combined score $>0.4$. Cytoscape software (V3.6.1) was used to visualize the PPI network. Disconnected nodes 
were excluded, the degree of centrality of the DEGsbaseline was calculated, and a submodule was clustered, as we previously described [16].

\section{Quantitative real-time quantitative PCR (qRT-PCR) and statistical analysis}

qRT-PCR was performed to measure the expression levels of DEGs using the $2^{-\Delta \Delta C q}$ method. $\beta$-Actin was used as the normalization control for mRNA. The design and synthesis of the primers and the qRT-PCR procedure were described previously [16]. The primers are listed in Additional file 1. Significant differences in expression levels were determined using $t$ test with a $P$ value $<0.05$. Statistical analysis was performed and visualized as we previously described [16].

\section{Results}

\section{Patients and leg-pain scores}

We enrolled 25 patients with sciatica aged between 19 and 54 years (mean age 40 years) who were seen in our department for integrated TCM treatment and 25 healthy volunteers aged between 19 and 30 years (mean age 23 years) as healthy controls. All patients underwent standard integrated TCM treatment for sciatica for at least 14 days.
The VAS score for pain in patients decreased $(P<0.000)$, from an average of $7.4 \pm 0.81$ at baseline to $2.1 \pm 0.9714$ days after integrated TCM treatment, indicating that all patients achieved clinical remission.

\section{Microarray analysis}

Microarray analysis identified 153 DEGs-baseline, which included 131 upregulated genes and 22 downregulated genes (Fig. 1). After integrated TCM treatment, 8 genes were differentially expressed in the peripheral blood of patients compared with those at baseline, and all of these genes were downregulated (Table 1). When we compared the DEGs-baseline and DEGs-TCM, we found that toll-like receptor 5 (TLR5), interleukin-1 receptor antagonist (IL1RN), and solute carrier family 8 member A1 (SLC8A1) were present in both groups (Table 1) and that TLR5, IL1RN, and SLC8A1 were upregulated in sciatica patients at baseline compared with healthy controls and were then downregulated in sciatica patients after integrated TCM treatment compared with baseline.

\section{Enrichment analysis of DEGs-baseline}

Enrichment analysis by Metascape revealed that upregulated genes at baseline were enriched in 115 GO BP, 25

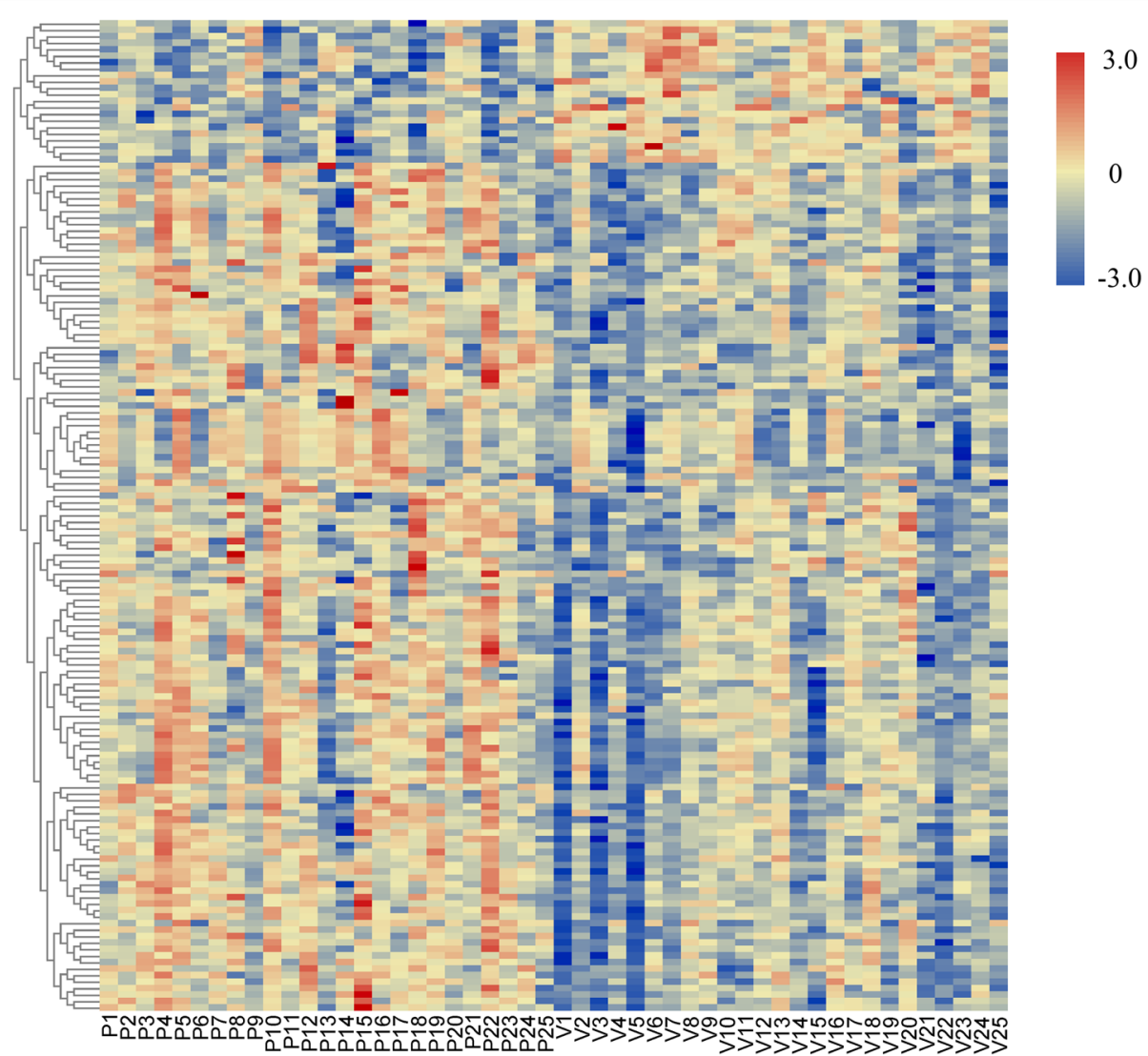

Fig. 1 Heatmap of the DEGs in the peripheral blood of patients with sciatica and healthy controls. The expression data were normalized using the $z$-score for indexes between -3 and 3 . Red, upregulated; blue, downregulated. $P$ patient with sciatica, $V$ healthy volunteer 
Table 1 Differentially expressed genes. After TCM treatment, 8 genes were differentially expressed in the peripheral blood of patients compared with those at baseline. TLR5, IL1RN, and SLC8A1 were upregulated in sciatica patients at baseline compared with healthy controls and were then downregulated in sciatica patients after TCM treatment compared with baseline.

\begin{tabular}{|c|c|c|c|c|c|}
\hline \multirow{2}{*}{$\begin{array}{l}\text { Gene } \\
\text { symbol }\end{array}$} & \multirow[t]{2}{*}{ Description } & \multicolumn{2}{|c|}{ Baseline vs. control } & \multicolumn{2}{|c|}{ TCM vs. Baseline } \\
\hline & & $P$ value & Fold change & $P$ value & Fold change \\
\hline TLR5 & Toll-like receptor 5 & 0.00 & 1.6 & 0.03 & 0.67 \\
\hline IL1RN & Interleukin 1 receptor antagonist & 0.01 & 1.7 & 0.03 & 0.64 \\
\hline SLC8A1 & Solute carrier family 8 member A1 & 0.00 & 1.5 & 0.04 & 0.63 \\
\hline RBM20 & RNA binding motif protein 20 & $>0.05$ & - & 0.03 & 0.62 \\
\hline GPER1 & G protein-coupled estrogen receptor 1 & $>0.05$ & - & 0.00 & 0.62 \\
\hline IL27 & Interleukin 27 & $>0.05$ & - & 0.01 & 0.59 \\
\hline SOCS1 & Suppressor of cytokine signalling 1 & $>0.05$ & - & 0.02 & 0.56 \\
\hline GRTP1-AS1 & GRTP1 antisense RNA 1 & $>0.05$ & - & 0.04 & 0.48 \\
\hline
\end{tabular}

TCM traditional Chinese medicine, TLR5 toll-like receptor 5, IL1RN interleukin 1 receptor antagonist, SLC8A1 solute carrier family 8 member A1, RBM20 RNA binding motif protein 20, GPER1 G protein-coupled estrogen receptor 1, IL27 interleukin 27, SOCS1 suppressor of cytokine signalling 1, GRTP1-AS1 GRTP1 antisense RNA 1

GO CC, 4 GO MF, and 6 KEGG pathways (Table 2). Downregulated genes at baseline were enriched in 10 GO BP, 1 GO MF, and 2 KEGG pathways (Table 3). Most of the enriched terms for both upregulated and downregulated DEGs at baseline were related to immunity and the inflammatory response. Enrichment analysis revealed immune and inflammatory response-related gene expression characteristics in the peripheral blood of patients with sciatica.

\section{PPI network}

The PPI network of DEGs-baseline consisted of 77 connected nodes and 255 edges (Fig. 2). To identify key genes in the PPI network, the plug-in CentiScaPe was used to calculate the centrality degree of each node. Genes with a high degree centrality included toll-like receptor (TLR4, degree 26), matrix metallopeptidase 9 (MMP9, degree 22), myeloperoxidase (MPO, degree 20), cathelicidin antimicrobial peptide (CAMP, degree 18), resistin (RETN, degree 18), TLR5 (degree, 17), etc. The top 10 genes with the highest degree centrality are listed in Table 4. MCODE analysis identified 1 cluster in the PPI network with threshold k-core $=5$. TLR4, MMP9, CAMP, and IL1RN were enriched in this cluster. The PPI network suggested that TLR4, MMP9, MPO, CAMP, RETN, TLR5, and IL1RN were key genes in the dysregulation of genes in the peripheral blood of patients with sciatica.

Table 2 Gene Ontology analysis of upregulated DEGs-baseline. The top 15 enriched terms of the upregulated genes in the peripheral blood of the patients with sciatica at baseline compared with the healthy controls according to the $P$ value. Log $10(P)$ is the $\log 10$-based $P$ value.

\begin{tabular}{llll}
\hline Term & Category & Description & Log10(P) \\
\hline GO:0002274 & GO BP & Myeloid leukocyte activation & -19.59 \\
GO:0002366 & GO BP & Leukocyte activation involved in immune response & -17.57 \\
GO:0002263 & GO BP & Cell activation involved in immune response & -17.51 \\
GO:0043312 & GO BP & Neutrophil degranulation & -17.42 \\
GO:0002283 & GO BP & Myeloid cell activation involved in immune response & -17.35 \\
GO:0002275 & GO BP & Neutrophil activation & -17.32 \\
GO:0042119 & GO BP & Neutrophil-mediated immunity & -17.15 \\
GO:0002446 & GO BP & Granulocyte activation & -17.12 \\
GO:0036230 & GO BP & Leukocyte degranulation & -17.02 \\
GO:0043299 & GO BP & Myeloid leukocyte-mediated immunity & -16.44 \\
GO:0002444 & GO BP & Regulated exocytosis & -16.10 \\
GO:0045055 & GO BP & Specific granule & -14.36 \\
GO:0042581 & GO CC & Tertiary granule & -14.34 \\
GO:0070820 & GO CC & Specific granule lumen & -12.84 \\
GO:0035580 & GO CC & - & -8.99 \\
\hline
\end{tabular}

GO gene ontology, BP biological processes, CC cellular components, MF molecular function 
Table 3 Gene Ontology analysis of downregulated DEGs-baseline. All enriched terms of the downregulated genes in the peripheral blood of the patients with sciatica at baseline compared with the healthy controls according to the $P$ value. $\log 10(P)$ is the log $10-$ based $P$ value.

\begin{tabular}{llll}
\hline Category & Term & Description & Log10(P) \\
\hline KEGG pathway & hsa04612 & Antigen processing and presentation & -4.34 \\
GO MF & GO:0030246 & Carbohydrate binding & -4.07 \\
KEGG pathway & hsa04650 & Natural killer cell-mediated cytotoxicity & -3.63 \\
GO BP & GO:0002708 & Positive regulation of lymphocyte-mediated immunity & -3.92 \\
GO BP & GO:0002705 & Positive regulation of leukocyte-mediated immunity & -3.62 \\
GO BP & GO:0002706 & Regulation of lymphocyte-mediated immunity & -3.46 \\
GO BP & GO:0002703 & Regulation of leukocyte-mediated immunity & -3.08 \\
GO BP & GO:0002699 & Positive regulation of immune effector process & -2.99 \\
GO BP & GO:0002449 & Lymphocyte-mediated immunity & -2.37 \\
GO BP & GO:0045089 & Positive regulation of innate immune response & -2.32 \\
GO BP & GO:0045088 & Regulation of innate immune response & -2.12 \\
GO BP & GO:0002697 & Regulation of immune effector process & -2.08 \\
GO BP & GO:0070997 & Neuron death & -2.40 \\
\hline GO gene ontogy
\end{tabular}

GO gene ontology, BP biological processes, CC cellular components, MF molecular function, KEGG Kyoto Encyclopedia of Genes and Genomes

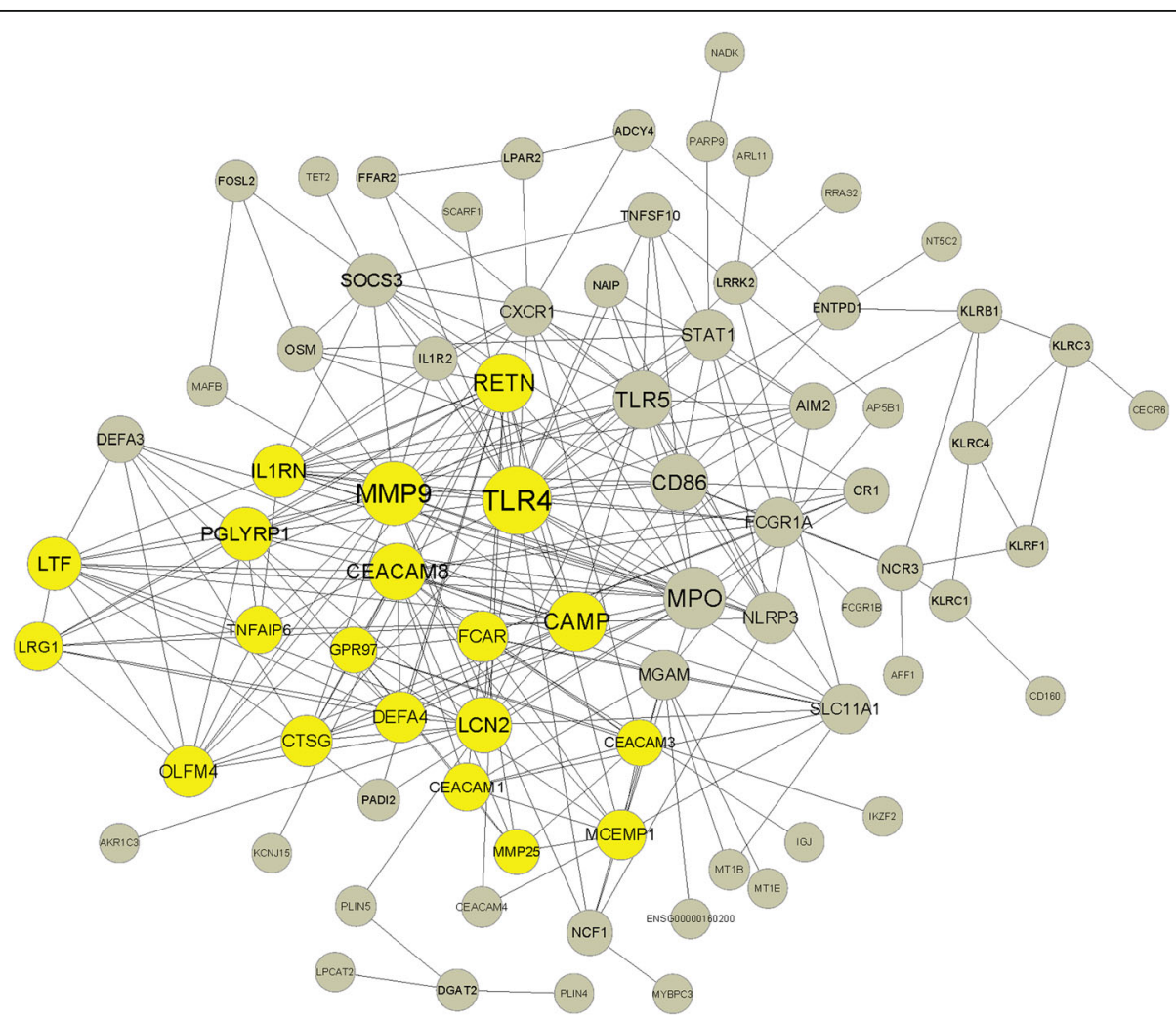

Fig. 2 PPI network of DEGs at baseline. Seventy-seven nodes with a combined score $>0.4$ and 255 edges were included. The size of the nodes represents the centrality degree. One cluster was identified by MCODE analysis with threshold k-core $=5$ and is colored yellow 
Table 4 Top 10 genes with the highest centrality degree in the PPI network. Centrality degree was calculated by the CentiScaPe plug-in, and one cluster was identified by MCODE analysis with threshold k-core $=5$

\begin{tabular}{lll}
\hline Gene & Degree & MCODE_cluster \\
\hline TLR4 & 26 & Clustered \\
MMP9 & 22 & Clustered \\
MPO & 20 & Unclustered \\
CAMP & 18 & Clustered \\
RETN & 18 & Clustered \\
TLR5 & 17 & Unclustered \\
CEACAM8 & 16 & Clustered \\
CD86 & 16 & Unclustered \\
LCN2 & 15 & Clustered \\
IL1RN & 13 & Clustered \\
\hline
\end{tabular}

TLR4 toll-like receptor 4, MMP9 matrix metallopeptidase 9, MPO myeloperoxidase, CAMP cathelicidin antimicrobial peptide, RETN resistin, TLR5 toll-like receptor 5, CEACAM8 CEA cell adhesion molecule 8, CD86 CD86 molecule, $L C N 2$ lipocalin 2, ILIRN interleukin 1 receptor antagonist

\section{Expression of DEGs}

We examined the expression levels of the key DEGsbaseline (TLR4, MMP9, MPO, CAMP, RETN, TLR5, and IL1RN) in the peripheral blood of patients at baseline and in the peripheral blood of healthy controls, as well as expression levels of DEGs-TCM in the peripheral blood of patients at baseline and in the peripheral blood of patients after integrated TCM treatment.

qRT-PCR showed that the expression levels of TLR4, MMP9, MPO, CAMP, RETN, TLR5, and IL1RN were increased in the peripheral blood of patients at baseline compared with the healthy controls (Fig. 3); the expression levels of TLR5, IL1RN, SLC8A1, RNA binding motif protein 20 (RBM20), G protein-coupled estrogen receptor 1 (GPER1), interleukin 27 (IL27), suppressor of cytokine signalling 1 (SOCS1), and GRTP1 antisense RNA 1
(GRTP1-AS1) were decreased in the peripheral blood of the patients after integrated TCM treatment compared with that at baseline (Fig. 4).

\section{Discussion}

With microarray analysis, we identified 153 DEGs in the peripheral blood of sciatica patients and healthy controls. Bioinformatic analysis revealed that these DEGs were mainly related to immunity and the inflammatory response and that TLR4, MMP9, MPO, CAMP, RETN, TLR5, and IL1R were the most important genes contributing to the dysregulation of these DEGs.

In the PPI network of DEGs-baseline, TLR4 had the highest centrality degree, which was consistent with our previous study [16]. TLR4 is the most studied Toll-like receptor because it plays a broad role in the inflammatory response [18]. It is also involved in innate neuroimmunity and neuropathy and mediates inflammatory and neuropathic pain [19]. An animal model confirmed that the expression of TLR4 in intervertebral discs and activation of TLR4 in intervertebral discs induced an inflammatory response, including the upregulation of tumor necrosis factor $\alpha$, IL-1 $\beta$, IL-6, and nitric oxide [20]. The inhibition of TLR4 in discs reduced inflammation and reversed pain-related neuroplasticity, suggesting that TLR4 is a potential target for treating disc-related inflammatory and neuropathic pain [21]. MMP9 is associated with sciatica in both peripheral blood and local discs. MMP9 has long been known to degrade collagen. It is expressed in intervertebral discs, which are full of collagen [22], and the expression levels of MMP9 decrease in free, protruded, and extruded discs [23]. MMP 9 is also involved in the activation and inactivation of inflammation, although the mechanism has not been completely elucidated [24]. Focal MMP9 causes leukocytes to migrate from peripheral blood to

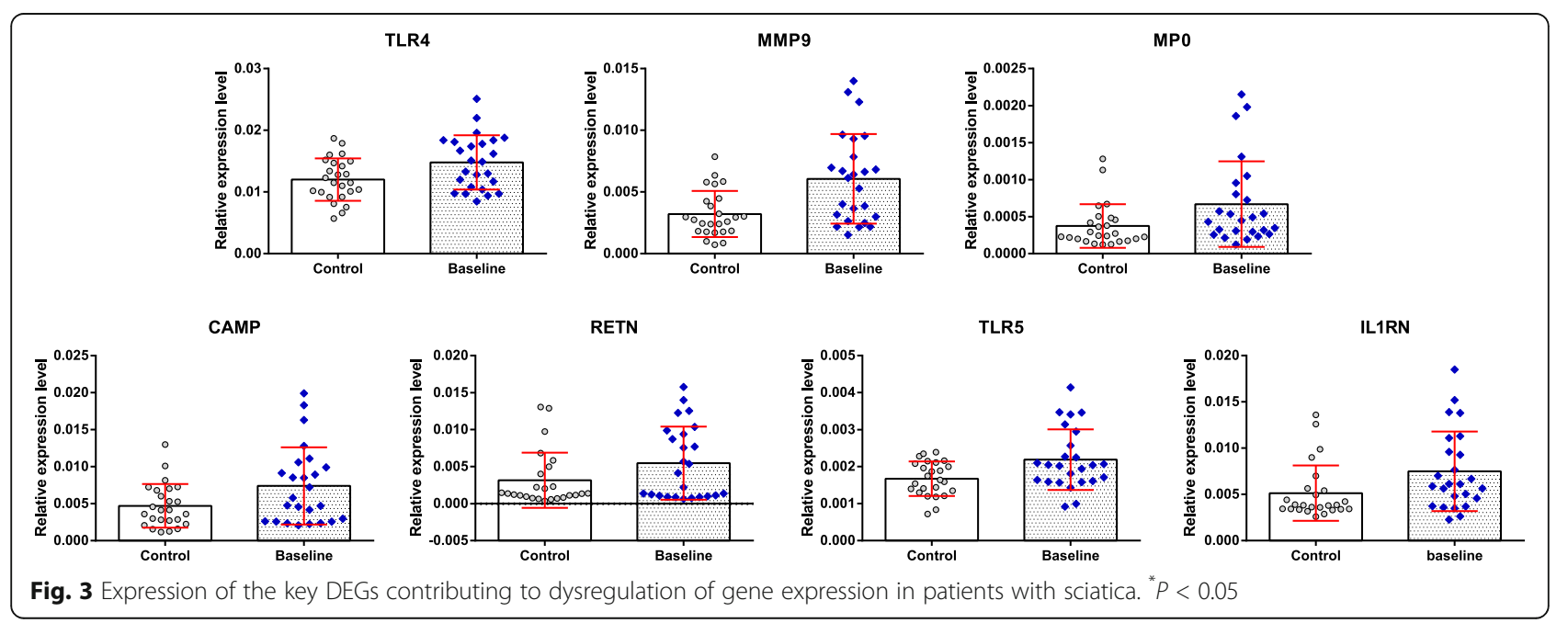



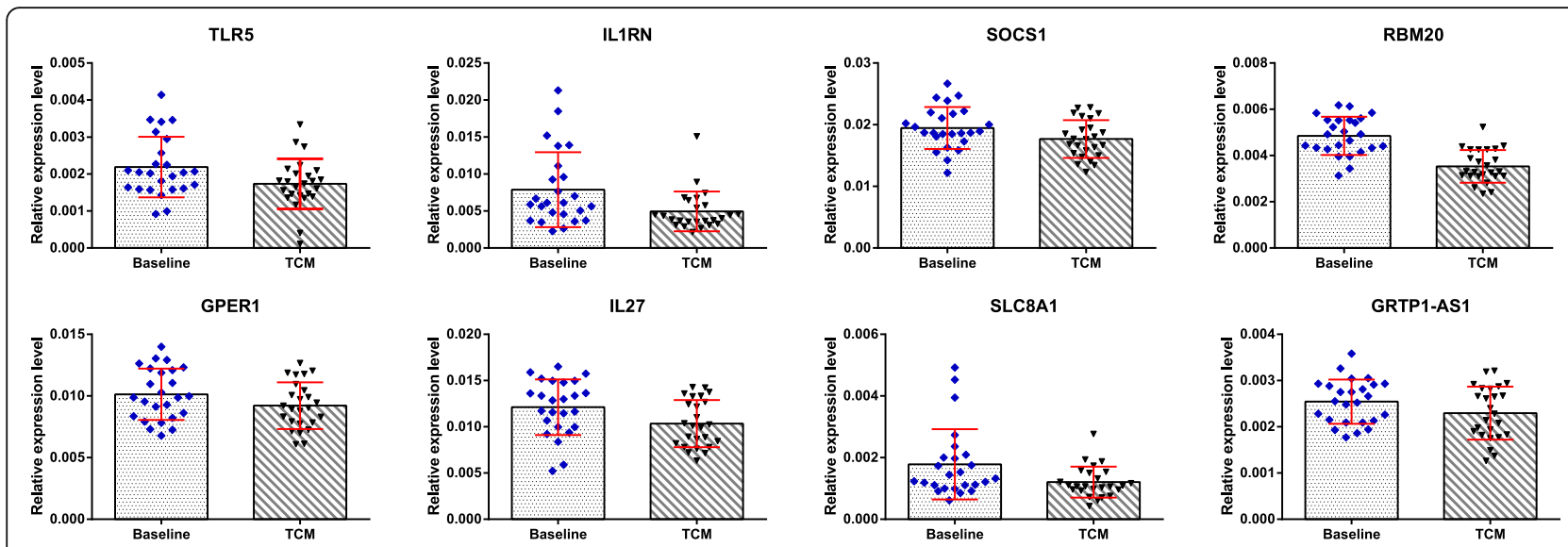

Fig. 4 Expression of DEGs of patients with sciatica after integrated TCM treatment compared with those at baseline. ${ }^{*} P<0.05$

tissues by promoting a chemotactic gradient, which has a role in the neuroinflammatory process [25]. MPO is mainly produced by circulating neutrophils and is linked to inflammatory conditions and degenerative neurological disorders. By producing hypochlorous acid-sphingomyelinase, which is released as a source of reactive oxygen species, MPO indirectly mediates inflammatory injury [26]. In stroke patients, MPO expression is increased in both the plasma and the serum [27]. The expression of MPO is increased in the brains of patients with Alzheimer's or Parkinson's disease [28, 29], and MPO has been reported to be activated in human multiple sclerosis plaques [30]. The exact mechanism of MPO in these neurological diseases is not completely clear. RETN levels were reported to be associated with inflammatory cytokines, including TNF- $\alpha$ receptor-2, IL-6, ICAM-1, lipoprotein-associated PLA2, and C-reactive protein $[31,32]$. RETN also upregulates TNF- $\alpha$ and IL-6 in human peripheral blood mononuclear cells as well as TNF- $\alpha$ and IL-12 in human macrophages [33, 34]. Combining these studies, a potential link between TLR4, MMP9, MPO, CAMP, RETN, and sciatica can be established, but microarray analysis showed that the expression of these genes was not changed after integrated TCM treatment. Integrated TCM treatment does not alleviate sciatica by regulating these genes.

After integrated TCM treatment, the pain of the patients was relieved, and the expression levels of TLR5, IL1RN, SLC8A1, RBM20, GPER1, IL27, SOCS1, and GRTP1-AS1 in peripheral blood were downregulated compared with baseline. We also found that TLR5, IL1RN, and SLC8A1 were upregulated in sciatica patients at baseline compared with healthy controls and downregulated after integrated TCM treatment compared with baseline, indicating that these genes played an important role in the pathogenesis and remission of sciatica. In the future, the expression of these genes can be considered as an objective indicator for evaluating the efficacy of integrated TCM in treating sciatica in the clinical setting.

Animal experiments have shown that the knockout or blockade of TLR5 can reduce pain $[35,36]$, which is consistent with our finding that TLR5 expression was downregulated in patients with pain relief after integrated TCM treatment. IL1RN polymorphism and genetic variability were reported to be associated with outcomes of intervetebral disc disease [37, 38], but no studies have elaborated the role of IL1RN mRNA expression in sciatica. The roles of SLC8A1, RBM20, GPER1, IL27, SOCS1, and GRTP1-AS1 in sciatica or in relieving sciatica are currently unknown. Future studies should further investigate the role of these genes in sciatica remission.

Our study had limitations. Some other factors, such as miRNAs and lncRNAs, are involved in the process of protein translation from mRNA, and protein expression is not only determined by mRNA. We did not perform western blotting to measure the protein levels of these genes, and the protein levels and mRNA levels of these genes may not be consistent. Changes in gene expression and alleviation of sciatica were simultaneously observed after integrated TCM treatment in the present study; whether integrated TCM treatment relieved sciatica by regulating the expression of these genes still needs further investigation.

\section{Conclusions}

In conclusion, we found that integrated TCM treatment alleviated sciatica while regulating the gene expression of TLR5, IL1RN, SLC8A1, RBM20, GPER1, IL27, SOCS1, and GRTP1-AS1 in the peripheral blood of patients. Our study provides new clues for studying the mechanism of TCM in treating sciatica. 


\section{Supplementary Information}

The online version contains supplementary material available at https://doi. org/10.1186/s13018-021-02280-1.

Additional file 1. Sequences of primers used for qRT-PCR

\section{Abbreviations}

TCM: Traditional Chinese medicine; DEGs: Differentially expressed genes; DEGs-baseline: DEGs in the peripheral blood of patients with sciatica and healthy controls; DEGs-TCM: DEGs in the peripheral blood of patients with sciatica after integrated TCM treatment compared with those at baseline; GO: Gene ontology; BP: Biological processes; CC: Cellular components; MF: Molecular functions; KEGG: Kyoto Encyclopedia of Genes and Genomes; PPI: Protein-protein interaction; qRT-PCR: Quantitative real-time quantitative PCR; TLR5: Toll-like receptor 5; IL1RN: Interleukin 1 receptor antagonist; SLC8A1: Solute carrier family 8 member A1; TLR4: Toll-like receptor; MMP9: Matrix metallopeptidase 9; MPO: Myeloperoxidase; CAMP: Cathelicidin antimicrobial peptide; RETN: Resistin; RBM20: RNA-binding motif protein 20; GPER1: G protein-coupled estrogen receptor 1; IL27: Interleukin 27; SOCS1: Suppressor of cytokine signalling 1; GRTP1-AS1: GRTP1 antisense RNA

\section{Acknowledgements}

Not applicable

\section{Authors' contributions}

Yi Wang: conceptualization, project administration, methodology, investigation, software, validation, formal analysis, writing-original draft, visualization, and funding acquisition. Guogang Dai and Yan Xu: supervision, project administration, resources, validation, funding acquisition, investigation, and writing — review \& editing. Ling Jiang and Zhibin Fu: methodology, software, validation, methodology, data curation, formal analysis, investigation, visualization, and writing —original draft. Jiao Xia and Guogang Tian: formal analysis, investigation, resources, data curation, and writing - original draft. Wanli Du: formal analysis, investigation, resources, data curation, and writing - original draft. All authors read and approved the final manuscript.

\section{Funding}

This study was supported by the Special Scientific Research Project of Orthopedics (Shang An Tong) of Sichuan Medical Association (2019SAT06), Sichuan Science and Technology Program (2018SZ0075), and Sichuan Provincial Administration of Traditional Chinese Medicine (2020JC0103). The funding bodies played no role in the design of the study and collection, analysis, and interpretation of data and in writing the manuscript.

\section{Availability of data and materials}

The gene expression datasets generated and analyzed during the current study are available in the GEO database (http://www.ncbi.nlm.nih.gov/geo) under the number GSE150408 and GSE124272. The datasets used and/or analyzed during the current study are available from the corresponding author on reasonable request.

\section{Ethics approval and consent to participate}

Ethical approval for this study was obtained from the Ethics Committee of the Sichuan Provincial Orthopedics Hospital. All participants provided informed written consent.

\section{Consent for publication}

Not applicable

\section{Competing interests}

The authors declare no conflict of interests.

\section{Author details}

${ }^{1}$ Cervicodynia/Omalgia/Lumbago/Sciatica Department 2, Sichuan Provincial Orthopedics Hospital, No. 132 West First Section First Ring Road, Wuhou District, Chengdu, Sichuan Province, China. ${ }^{2}$ Experiment Teaching Center for Preclinical Medicine, Chengdu Medical College, No. 783, Xindu Avenue, Xindu District, Chengdu, Sichuan Province, China. ${ }^{3}$ College Hospital, Sichuan
Agricultural University, Chengdu Campus, No. 211 Huiming Road, Wenjiang District, Chengdu, Sichuan Province, China. ${ }^{4}$ Department of Lower Extremities, Sport Hospital Affiliated to Chengdu Sport Institute, No. 2, Tiyuan Road, Wuhou District, Chengdu, Sichuan Province, China.

Received: 17 January 2021 Accepted: 3 February 2021

Published online: 11 February 2021

\section{References}

1. Stafford MA, Peng P, Hill DA. Sciatica: a review of history, epidemiology, pathogenesis, and the role of epidural steroid injection in management. $\mathrm{Br}$ J Anaesth. 2007;99(4):461-73.

2. Valat JP, Genevay S, Marty M, Rozenberg S, Koes B. Sciatica. Best Pract Res Clin Rheumatol. 2010;24(2):241-52.

3. Wang $Y$, Jiang $L$, Dai G, Li S, Mu X. Bioinformatics analysis reveals different gene expression patterns in the annulus fibrosis and nucleus pulpous during intervertebral disc degeneration. Exp Ther Med. 2018;16(6):5031-40.

4. Zhu Z, Chen G, Jiao W, Wang D, Cao Y, Zhang Q, et al. Identification of critical genes in nucleus pulposus cells isolated from degenerated intervertebral discs using bioinformatics analysis. Mol Med Rep. 2017;16(1): 553-64.

5. Guo W, Zhang B, Li Y, Duan HQ, Sun C, Xu YQ, et al. Gene expression profile identifies potential biomarkers for human intervertebral disc degeneration. Mol Med Rep. 2017;16(6):8665-72.

6. Xu F, Gao F, Liu Y, Wang Z, Zhuang X, Qu Z, et al. Bioinformatics analysis of molecular mechanisms involved in intervertebral disc degeneration induced by TNF-alpha and IL-1beta. Mol Med Rep. 2016;13(3):2925-31.

7. Purmessur D, Walter BA, Roughley PJ, Laudier DM, Hecht AC, latridis J. A role for TNFalpha in intervertebral disc degeneration: a non-recoverable catabolic shift. Biochem Biophys Res Commun. 2013;433(1):151-6.

8. Matsui $Y$, Maeda M, Nakagami W, Iwata $\mathrm{H}$. The involvement of matrix metalloproteinases and inflammation in lumbar disc herniation. Spine (Phila Pa 1976). 1998:23(8):863-8 discussion 8-9.

9. Shamji MF, Setton LA, Jarvis W, So S, Chen J, Jing L, et al. Proinflammatory cytokine expression profile in degenerated and herniated human intervertebral disc tissues. Arthritis Rheum. 2010;62(7):1974-82.

10. McCarron RF, Wimpee MW, Hudkins PG, Laros GS. The inflammatory effect of nucleus pulposus. A possible element in the pathogenesis of low-back pain. Spine (Phila Pa 1976). 1987;12(8):760-4

11. Bailey CS, Rasoulinejad P, Taylor D, Sequeira K, Miller T, Watson J, et al. Surgery versus conservative care for persistent sciatica lasting 4 to 12 months. N Engl J Med. 2020;382(12):1093-102.

12. Mo Z, Li D, Zhang R, Chang M, Yang B, Tang S. Comparisons of the effectiveness and safety of tuina, acupuncture, traction, and Chinese herbs for lumbar disc herniation: a systematic review and network meta-analysis. Evid Based Complement Alternat Med. 2019;2019:6821310.

13. Tang S, Mo Z, Zhang R. Acupuncture for lumbar disc herniation: a systematic review and meta-analysis. Acupunct Med. 2018;36(2):62-70.

14. Long $Y$. Treatment of protrusion of the lumbar intervertebral disc by TCM massage. J Tradit Chin Med. 2002;22(2):126-7.

15. Kreiner DS, Hwang SW, Easa JE, Resnick DK, Baisden JL, Bess S, et al. An evidence-based clinical guideline for the diagnosis and treatment of lumbar disc herniation with radiculopathy. Spine J. 2014;14(1):180-91.

16. Wang Y, Dai G, Li L, Liu L, Jiang L, Li S, et al. Transcriptome signatures reveal candidate key genes in the whole blood of patients with lumbar disc prolapse. Exp Ther Med. 2019;18(6):4591-602.

17. Zhou Y, Zhou B, Pache L, Chang M, Khodabakhshi AH, Tanaseichuk O, et al. Metascape provides a biologist-oriented resource for the analysis of systems-level datasets. Nat Commun. 2019;10(1):1523.

18. Lacagnina MJ, Watkins LR, Grace PM. Toll-like receptors and their role in persistent pain. Pharmacol Ther. 2018;184:145-58.

19. Ji RR, Chamessian A, Zhang YQ. Pain regulation by non-neuronal cells and inflammation. Science. 2016:354(6312):572-7.

20. Rajan NE, Bloom O, Maidhof R, Stetson N, Sherry B, Levine M, et al. Toll-like receptor 4 (TLR4) expression and stimulation in a model of intervertebral disc inflammation and degeneration. Spine (Phila Pa 1976). 2013;38(16): 1343-51.

21. Krock E, Millecamps M, Currie JB, Stone LS, Haglund L. Low back pain and disc degeneration are decreased following chronic toll-like receptor 4 inhibition in a mouse model. Osteoarthr Cartil. 2018;26(9):1236-46. 
22. Basaran R, Senol M, Ozkanli S, Efendioglu M, Kaner T. Correlation of matrix metalloproteinase (MMP)-1, -2, -3, and -9 expressions with demographic and radiological features in primary lumbar intervertebral disc disease. J Clin Neurosci. 2017;41:46-9.

23. Li PB, Tang WJ, Wang K, Zou K, Che B. Expressions of IL-1alpha and MMP-9 in degenerated lumbar disc tissues and their clinical significance. Eur Rev Med Pharmacol Sci. 2017;21(18):4007-13.

24. Parks WC, Wilson CL, Lopez-Boado YS. Matrix metalloproteinases as modulators of inflammation and innate immunity. Nat Rev Immunol. 2004; 4(8):617-29.

25. Hannocks MJ, Zhang X, Gerwien H, Chashchina A, Burmeister M, Korpos E, et al. The gelatinases, MMP-2 and MMP-9, as fine tuners of neuroinflammatory processes. Matrix Biol. 2019;75-76:102-13.

26. Pravalika K, Sarmah D, Kaur H, Wanve M, Saraf J, Kalia K, et al. Myeloperoxidase and neurological disorder: a crosstalk. ACS Chem Neurosci. 2018;9(3):421-30.

27. Tay A, Tamam Y, Yokus B, Ustundag M, Orak M. Serum myeloperoxidase levels in predicting the severity of stroke and mortality in acute ischemic stroke patients. Eur Rev Med Pharmacol Sci. 2015;19(11):1983-8.

28. Green PS, Mendez AJ, Jacob JS, Crowley JR, Growdon W, Hyman BT, et al. Neuronal expression of myeloperoxidase is increased in Alzheimer's disease. J Neurochem. 2004;90(3):724-33.

29. Gellhaar S, Sunnemark D, Eriksson H, Olson L, Galter D. Myeloperoxidaseimmunoreactive cells are significantly increased in brain areas affected by neurodegeneration in Parkinson's and Alzheimer's disease. Cell Tissue Res. 2017;369(3):445-54.

30. Sospedra M, Martin R. Immunology of multiple sclerosis. Semin Neurol. 2016;36(2):115-27.

31. Pang SS, Le YY. Role of resistin in inflammation and inflammation-related diseases. Cell Mol Immunol. 2006;3(1):29-34.

32. Filkova M, Haluzik M, Gay S, Senolt L. The role of resistin as a regulator of inflammation: implications for various human pathologies. Clin Immunol. 2009:133(2):157-70.

33. Silswal N, Singh AK, Aruna B, Mukhopadhyay S, Ghosh S, Ehtesham NZ. Human resistin stimulates the pro-inflammatory cytokines TNF-alpha and IL12 in macrophages by NF-kappaB-dependent pathway. Biochem Biophys Res Commun. 2005;334(4):1092-101.

34. Bokarewa M, Nagaev I, Dahlberg L, Smith U, Tarkowski A. Resistin, an adipokine with potent proinflammatory properties. J Immunol. 2005;174(9): 5789-95.

35. Stokes JA, Cheung J, Eddinger K, Corr M, Yaksh TL. Toll-like receptor signaling adapter proteins govern spread of neuropathic pain and recovery following nerve injury in male mice. J Neuroinflammation. 2013;10:148.

36. Das N, Dewan V, Grace PM, Gunn RJ, Tamura R, Tzarum N, et al. HMGB1 activates proinflammatory signaling via TLR5 leading to allodynia. Cell Rep. 2016;17(4):1128-40.

37. Kim DH, Lee SH, Kim KT, Yu SD. Association of interleukin-1 receptor antagonist gene polymorphism with response to conservative treatment of lumbar herniated nucleus pulposus. Spine (Phila Pa 1976). 2010;35(16):152731.

38. Moen A, Schistad El, Rygh LI, Roe C, Gjerstad J. Role of IL1A rs1800587, IL1B rs 1143627 and IL1RN rs2234677 genotype regarding development of chronic lumbar radicular pain; a prospective one-year study. PLoS One. 2014;9(9):e107301.

\section{Publisher's Note}

Springer Nature remains neutral with regard to jurisdictional claims in published maps and institutional affiliations.

\section{Ready to submit your research? Choose BMC and benefit from:}

- fast, convenient online submission

- thorough peer review by experienced researchers in your field

- rapid publication on acceptance

- support for research data, including large and complex data types

- gold Open Access which fosters wider collaboration and increased citations

- maximum visibility for your research: over $100 \mathrm{M}$ website views per year

At BMC, research is always in progress.

Learn more biomedcentral.com/submissions 